Boletín de la Sociedad Geológica Mexicana

VOLUMEN 62, NÚM. 2, 2010, P. 213-220

\title{
Ecuación para la Corrección Poynting en Termodinámica de Equilibrio de Fases Gases no Polares-Sistemas Acuosos. Aplicación al Sistema $\mathrm{H}_{2} \mathrm{~S}-\mathrm{H}_{2} \mathrm{O}-\mathrm{NaCl}$
}

\author{
José Martínez Reyes ${ }^{1, *}$, Renee J. Pérez ${ }^{2,3}$, Eduardo González Partida ${ }^{1}$, \\ Jorge A. Tinoco Michel ${ }^{1}$ \\ ${ }^{1}$ Centro de Geociencias, Universidad Nacional Autónoma de México Campo de Juriquilla, Qro., México, apartado postal 76230. \\ ${ }^{2}$ Department of Chemical and Petroleum Engineering, University of Calgary, 500 University Drive, Calgary Alberta, Canada, T2N \\ $1 \mathrm{~N} 4$. \\ ${ }^{3}$ ExxonMobil Upstream Research Company 3120 Buffalo Speedway, Houston, TX 77098. \\ *jmreyes@geociencias.unam.mx
}

\section{Resumen}

En el presente trabajo se empleó la expresión semi-teórica del volumen molar parcial a dilución infinita acuosa para solutos volátiles no electrolíticos $\left(V_{2}^{0}\right)$, desarrollada por Plyasunov et al. (2000b) con el propósito de proponer una ecuación novedosa para corrección Poynting. La fórmula matemática de $V_{2}^{0}$ considera la densidad y la compresibilidad isotérmica del solvente como variables, así como el segundo coeficiente virial cruzado solvente-soluto $\left(\beta_{12}\right)$ y el segundo coeficiente virial del solvente puro $\left(\beta_{11}\right)$.

La ecuación se integró analíticamente respecto a la presión utilizando algunas correlaciones auxiliares que se encuentran en la literatura, para obtener así la expresión matemática del incremento isotérmico de energía estándar (o potencial químico) de Gibbs del soluto a dilución infinita $\left(\Delta G_{2}{ }^{0}\right)$. La ecuación matemática es aplicable a los solutos cuyo $\beta_{12}$ es conocido o se puede estimar, en un intervalo de temperatura de $273.16 \mathrm{~K}$ a $647 \mathrm{~K}$, valores de presión de hasta 2 kbar y en salmueras con fuerza iónica de hasta $6 \mathrm{~m} \mathrm{NaCl}$. La expresión proporciona excelente correlación con datos experimentales, tal como se demuestra para el sistema $\mathrm{H}_{2} \mathrm{~S}-\mathrm{H}_{2} \mathrm{O}-\mathrm{NaCl}(\mathrm{con}$ desviación máxima del 7\%), mediante un modelo termodinámico que emplea esta fórmula propuesta, acoplada a la Ley de Henry y a la ecuación de estado de Soave-Redlich-Kwong para describir el equilibrio de fases líquido-vapor. De igual manera, se propone una expresión alternativa para calculo de $V_{2}^{0}$ (con valores semejantes de desviación máxima).

Palabras clave: corrección Poynting, modelado termodinámico.

\begin{abstract}
In this paper we used the semitheoretical expression for the partial molar volume at infinite dilution of volatile aqueous non-electrolyte solute $\left(V_{2}^{0}\right)$, developed by Plyasunov et al. (2000b) in order to propose a new equation for Poynting correction. The mathematical formula $V_{2}^{0}$ considers as variables the density and isothermal compressibility of the solvent, as well as the second cross solvent-solute virial coefficient $\left(\beta_{12}\right)$ and the second virial coefficient of pure solvent $\left(\beta_{11}\right)$.

The equation was integrated analytically with respect to pressure using some auxiliary correlations found in the literature, and thereby obtain a mathematical expression of the isothermal pressure increment of the standard (infinite dilution) Gibbs energy (or chemical potential) of the solute $\left(\Delta G_{2}{ }^{0}\right)$. The mathematical equation is applicable to solutes whose $\beta_{12}$ is known or can be estimated, in a temperature range of $273.16 \mathrm{~K}$ to $647 \mathrm{~K}$, values of pressure up to $2 \mathrm{kbar}$ and brines with ionic strength equal to $6 \mathrm{~m} \mathrm{NaCl}$. The expression fits the experimental data very well, as shown for the $\mathrm{H}_{2} \mathrm{~S}-\mathrm{H}_{2} \mathrm{O}-\mathrm{NaCl}$ system (with maximum deviation of 7\%), through a thermodynamic model that uses this formula proposal coupled with the Law of Henry and the Soave-Redlich-Kwong equation of state
\end{abstract}


for modeling the liquid-vapor phase equilibria. Similarly, we propose an alternative expression for calculating $V_{2}^{0}$ (with similar values of maximum deviation).

Keywords: thermodynamic modeling, Poynting correction.

\section{Introducción}

La energía estándar de Gibbs molar parcial de hidratación de no electrolitos $\left(\Delta G_{2}{ }^{\circ}\right)$ es la propiedad termodinámica más importante para modelado geoquímico (Plyasunov et al., 2000b), debido a que con ella se aplica la corrección Poynting en termodinámica de equilibrio de fases líquidovapor a temperaturas y presiones altas (Carroll, 1991; Plyasunov et al., 2000a, b; Fernández-Prini et al., 2003).

En el presente trabajo se ha considerado la expresión semi-teórica para el cálculo del volumen molar parcial a dilución infinita para solutos volátiles acuosos no electrolíticos $\left(V_{2}^{0}\right)$, desarrollada por Plyasunov et al. (2000a, b) con el objeto de proponer una ecuación para corrección Poynting, así como una fórmula alternativa para cálculo de $V_{2}^{0}$.

La fórmula para determinación de $V_{2}^{0}$ de Plyasunov et al. $(2000 \mathrm{a}, \mathrm{b})$ fue desarrollada con base en la teoría de fluctuación de una solución (Kirkwood y Buff, 1951) así como de la teoría de fluctuación mecánica estadística de una solución (O’Connell, 1971, 1994 y 1995). La ecuación considera la densidad y compresibilidad isotérmica del solvente, así como el segundo coeficiente virial cruzado solvente-soluto y el segundo coeficiente virial del solvente puro, lo que permite aplicarla en un amplio rango de temperatura y presión. Esta ecuación es la siguiente (Plyasunov et al., 2000a, b):

$$
\begin{aligned}
V_{2}^{0}= & N V_{1}^{0}+\kappa_{P T} R T(1-N)+2 \kappa_{P, T} R T \rho_{P, T} \Omega\left(\beta_{12}-N \beta_{11}\right) e^{-C_{1} \rho_{P, T}} \\
& +\left(\frac{a}{T^{5}}+b\right)\left(e^{C_{2} \rho_{P, T}}-1\right)
\end{aligned}
$$

Donde $V_{1}^{0} \mathrm{y} \kappa_{P T}$ son el volumen molary la compresibilidad isotérmica del agua en unidades de $\mathrm{cm}^{3} \mathrm{~mol}^{-1} \mathrm{y} \mathrm{bar}^{-1}$, respectivamente; $\rho_{P, T}$ es la densidad del agua a presión y temperatura determinada expresada en $\mathrm{m}^{3} / \mathrm{kg} ; \Omega$ es un factor de conversión igual a 1000/18.016 mol $/ \mathrm{kg} \mathrm{H}_{2} \mathrm{O} ; c_{1}=0.0033 \mathrm{y}$ $c_{2}=0.002$, ambas en $\mathrm{m}^{3} / \mathrm{kg}$, se pueden considerar constantes universales; $N=1.53+/-0.05$ (adimensional); $a=-2.8+/$ $0.04 \mathrm{~m}^{3} \mathrm{~K}^{5} \mathrm{~kg}^{-1}$ y $b=1.23+/-0.03 \mathrm{~m}^{3} \mathrm{~kg}^{-1}$, son parámetros específicos del soluto y se encuentran tabulados o se dan estrategias de cálculo para los mismos en Plyasunov et al. $(2000 \mathrm{a}, \mathrm{b}) ; \beta_{12}$ es el segundo coeficiente virial cruzado solvente-soluto y $\beta_{11}$ es el segundo coeficiente virial de agua pura en $\mathrm{m}^{3} / \mathrm{mol}$.

Estos coeficientes viriales pueden calcularse a partir de la expresión de Pitzer (1995):

$$
\beta_{i j}=\frac{2 \pi \sigma_{i j}^{3} N_{A v}}{3}\left\{1-\left(\lambda^{3}-1\right)\left[e^{\frac{\varepsilon_{i j}}{(T) \kappa_{B}}}-1\right]\right\}
$$

donde $\sigma_{i j}=2.56 \times 10^{-10} \mathrm{~m}$ es la distancia entre los centros de masa en las colisiones de las partículas $i$ y $j, \lambda=1.22$ (se considera una constante universal) es el ancho del pozo potencial en diámetros moleculares, $N_{A \nu}=6.023 \times 10^{23}$ es el número de Avogadro, $\varepsilon_{i j} / \kappa_{B}=1255 \mathrm{~K}$ es la profundidad del pozo, $\varepsilon_{i j}$ representa la energía potencial mínima entre las partículas $i$ y $j$ y $\kappa_{B}$ es el factor de Boltzmann (Plyasunov et al., 2000a, b).

El cambio isotérmico de energía estándar de Gibbs (o potencial químico) del soluto volátil $\left(\Delta G_{2}{ }^{0}\right)$, se puede obtener integrando analíticamente la ecuación (1) respecto a la presión $(P)$ asumiendo así de manera rigurosa la dependencia matemática que guarda $V_{2}^{0}$ respecto a $P$ (Fernández-Prini et al., 2003), de acuerdo a la expresión (Plyasunov et al., 2000a, b; Fernández-Prini et al., 2003):

$$
\Delta G_{2}^{0}=\Delta G_{2}^{0}(T, P)-\Delta G_{2}^{0}\left(T, P_{\text {sat }}\right)=\mu_{i b}^{0}-\mu_{i b}^{P_{s a t}}=\int_{P_{\text {sat }}}^{P} V_{2}^{0} d P
$$

Con $\Delta G_{2}{ }^{0}$ se aplica la corrección Poynting, para compensar la desviación del potencial químico del soluto gaseoso en la fase líquida a temperatura y presión altas (Carroll, 1991; Orbey y Sandler, 1998; Fernández-Prini et al., 2003; Pérez et al., 2009).

\section{Integración Analítica de $V_{2}^{0}$}

En la integración de la ecuación (1), se asumió que $V_{1}^{0}$, $\kappa_{P, T}$ y $\rho_{P, T}$ también dependen de la presión. Para introducir esta dependencia se emplearon una serie de correlaciones auxiliares. En primer lugar, la compresibilidad del solvente se calculó de acuerdo a:

$$
\kappa_{P T}=-\frac{1}{V}\left(\frac{\partial V}{\partial P}\right)_{T}=\frac{A}{P+B}
$$

La ecuación (4) describe la curva de presión de vapor saturado de agua bajo su punto critico (Rowlinson y Swinton, 1982; Prausnitz et al., 1999; Plyasunov et al., 2000b), lo cual es la condición limitante de la expresión desarrollada en este trabajo, donde $B=3430$ bar (Heidemann et al., 1984) y $A$ se puede calcular de acuerdo a la fórmula de Kell (1975): 


$$
A=3431 \kappa(T, 1 \text { bar })
$$

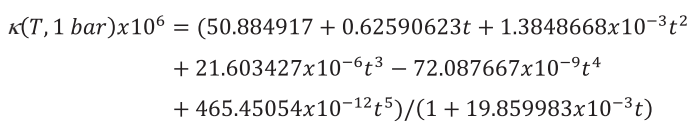

Donde $t$ es la temperatura expresada en grados Celsius.

A partir de la ecuación (4) se obtienen también las siguientes expresiones para el volumen molar parcial y la densidad del solvente:

$$
\begin{gathered}
V_{1}^{0}=v_{0}\left[\frac{P_{\text {sat }}+B}{P+B}\right]^{A} \\
\rho_{P, T}=\frac{1}{\Omega v_{0}}\left[\frac{P+B}{P_{\text {sat }}+B}\right]^{A}
\end{gathered}
$$

Donde la presión de vapor saturado $P_{\text {sat }}$ a una determinada $T$ se obtiene a partir de Saul y Wagner (1987), mientras que el volumen específico de agua $v_{0}$ a su presión de vapor saturado $P_{\text {sat }}$, se calcula a partir de Haas (1970).

Al sustituir las ecuaciones (2), (4)-(7) en la (1) se obtiene la expresión matemática del volumen molar parcial a dilución infinita acuosa para solutos volátiles no electrolíticos como función explícita de la presión.

Para facilitar la integración analítica de la ecuación (3), esta se divide en cuatro expresiones integrales simples:

$$
\Delta G_{2}^{0}=\int_{P_{\text {sat }}}^{P} V_{2}^{0} d P=\Delta G_{21}^{0}(A)+\Delta G_{22}^{0}(B)+\Delta G_{23}^{0}(C)+\Delta G_{24}^{0}(D)
$$

El desglose de esta integral (el desarrollo algebraico detallado se muestra en los Anexos correspondientes), es el siguiente:

$$
\begin{gathered}
\Delta G_{21}^{0}=\int_{P_{\text {sat }}}^{P} N V_{1}^{0} d P=\frac{N v_{0}\left(P_{\text {sat }}+B\right)^{A}}{1-A}\left[\frac{1}{(P+B)^{A-1}}-\frac{1}{\left(P_{\text {sat }}+B\right)^{A-1}}\right] \\
\Delta G_{22}^{0}=\int_{P_{\text {sat }}}^{P} \kappa_{P, T} R T(1-N) d P=R T(1-N) \ln \left[\frac{P+B}{P_{\text {sat }}+B}\right]^{A} \\
\Delta G_{23}^{0}=\int_{P_{\text {sat }}}^{P} 2 \kappa_{P, T} R T \rho_{P, T} \Omega\left(\beta_{12}-N \beta_{11}\right) e^{-C_{1} \rho_{P, T}} d P \\
\Delta G_{23}^{0}=2 R T \Omega\left(\beta_{12}-N \beta_{11}\right)\left(\frac{1}{C_{1}}\right)\left\{\exp \left[-\frac{C_{1}}{v_{0}}\right]-\exp \left[-C_{1} \rho_{P, T}\right]\right\} \\
\Delta G_{24}^{0}=\int_{P_{\text {sat }}}^{P}\left(\frac{a}{T^{5}}+b\right)\left(e^{\left.C_{2} \rho_{P, T}-1\right) d P}\right.
\end{gathered}
$$

Se debe notar que el termino $\Delta G_{24}{ }^{0}$ (Ecuación 9d) se ha obtenido aproximando la función exponencial como un polinomio de grado 4 (ver Anexo B). Como ya se mencionó, esta expresión es función de la densidad y la compresibilidad isotérmica del solvente, así como del segundo coeficiente virial cruzado solvente-soluto, del segundo coeficiente virial del solvente, de la temperatura y de la presión del sistema. Esta ecuación es aplicable en un intervalo de temperatura de $273.16 \mathrm{~K}$ a $647 \mathrm{~K}$, valores de presión de hasta 2 kbar y en salmueras con fuerza iónica de hasta $6 \mathrm{~m} \mathrm{NaCl}$. La expresión obtenida permite cálculos más precisos de equilibrio de fases líquido-vapor, a través de un cálculo exacto del factor de corrección Poynting (Carroll, 1991; Fernández-Prini et al., 2003).

Para estudiar el impacto de incluir o no la dependencia del volumen molar sobre la presión en el cálculo de equilibrio termodinámico de fases, la presente ecuación se empleó para determinar el equilibrio del sistema $\mathrm{H}_{2} \mathrm{~S}_{-} \mathrm{H}_{2} \mathrm{O}-$ $\mathrm{NaCl}$, tal como se describe a continuación

\section{Modelo Termodinámico con Integración Simple de $V_{2}^{0}$}

Para calcular el equilibrio del sistema $\mathrm{H}_{2} \mathrm{~S}-\mathrm{H}_{2} \mathrm{O}-\mathrm{NaCl}$ se ha considerado un modelo termodinámico que describe el comportamiento de la mezcla de gases en agua y en salmuera, como la de cloruro de sodio. El primer punto para el análisis termodinámico del equilibrio de fases es la igualdad de potenciales químicos $(\mu)$ de cada componente que coexiste entre las fases (Fernández-Prini y Crovetto, 1989; Carroll, 1991; Prausnitz et al., 1999; Orbey y Sandler, 1998; Fernández-Prini et al., 2003). La condición de equilibrio para un soluto gaseoso 2 entre una fase vapor $(v)$ y una salmuera $(b)$ puede escribirse de la siguiente manera:

$$
\mu_{2 v}=\mu_{2 b}
$$

En el modelo, el potencial químico del soluto en la salmuera se relaciona con el potencial químico del soluto en agua pura a través de los coeficientes “salting-out", definidos por Setchenow (1892). Los coeficientes de fugacidad de los componentes de la mezcla de vapor se pueden calcular con cualquier ecuación de estado. En este trabajo se empleó la ecuación de estado de Soave-Redlich-Kwong (Soave, 1972). El parámetro de interacción binaria del $\mathrm{H}_{2} \mathrm{~S}-\mathrm{H}_{2} \mathrm{O}$ 
resultante es $k_{i j}=0.20$. Para la fase de salmuera, la fugacidad de los solutos volátiles se calcula con la ley de Henry modificada (Harvey, 1996; Fernández-Prini y Crovetto, 1989; Fernández-Prini et al., 2003; Pérez y Heidemann, 2006; Pérez et al., 2009).

Para el sistema en estudio, la ecuación (10) se puede expresar como:

$$
f_{2 v}=H_{2 w} \gamma_{2 b} \chi_{2 b} \exp \left(S_{2} m_{k}\right) \exp \int_{P_{\text {sat }}}^{P} \frac{V_{2}^{0}(P, T) d P}{R T}
$$

donde $f_{2 v}$ es la fugacidad del soluto gaseoso en la fase vapor, $H_{2 w}$ la constante de Henry del soluto gaseoso en el agua, $\gamma_{2 b}$ el coeficiente de actividad del soluto gaseoso en la salmuera, $\chi_{2 b}$ la fracción molar del soluto gaseoso en la salmuera, $S_{2}$ el coeficiente "salting-out" o de Setchenow del soluto gaseoso, $m_{k}$ la molalidad de la sal $k$ disuelta en la salmuera, $V_{2}^{0}$ el volumen molar parcial a dilución infinita acuosa del soluto volátil no electrolítico, $P$ la presión del sistema, $P_{\text {sat }}$ la presión de saturación de la salmuera, $R$ la constante de los gases y $T$ la temperatura absoluta del sistema. El término exponencial en el lado derecho de la ecuación (11) es el factor de corrección Poynting (Carroll, 1991; Fernández-Prini et al., 2003).

Habitualmente, para facilitar su cálculo se asume un valor medio de $V_{2}^{0}$ en el rango de presiones de interés, lo cual resulta en la siguiente expresión (Carroll, 1991; Pérez et al., 2009):

$$
\ln \left(f_{2 v}\right)=\ln \left(H_{2 w} \gamma_{2 b} \chi_{2 b}\right)+\left(S_{2} m_{k}\right)+\left[V_{2}^{0}\left(P-P_{s a t}\right)\right] / R T
$$

Además:

$$
f_{2 v}=\varphi_{2 v} y_{2} P
$$

donde $\varphi_{2 v}$ es el coeficiente de fugacidad del soluto volátil en la fase vapor e $y_{2}$ la fracción molar del soluto volátil en la fase vapor.

Las constantes de Henry del $\mathrm{H}_{2} \mathrm{~S}$ en $\mathrm{H}_{2} \mathrm{O}$ se obtuvieron directamente de Fernández-Prini et al. (2003) y Harvey (1996), con la expresión:

$$
\ln \left(\frac{H_{i w}}{p_{1}^{*}}\right)=\frac{A}{T_{R}}+B \frac{\tau^{0.355}}{T_{R}}+C T_{R}^{-0.41} \exp (\tau)
$$

donde $\tau=1-T_{R}, T_{R}=T / T_{C l}, T_{C l}$ es la temperatura crítica del solvente aceptada por IAPWS (1995) (647.096 K para el $\mathrm{H}_{2} \mathrm{O}$ ), $A=-4.51499, B=5.23538, C=4.42126$ ( son constantes especificas del $\mathrm{H}_{2} \mathrm{~S}$ ) y $p_{1}{ }^{*}$ es la presión de vapor del solvente a la temperatura de interés. $p_{1}{ }^{*}$ se calcula a partir de la correlación de Wagner y Pruss (1993) para el $\mathrm{H}_{2} \mathrm{O}$, con la expresión:

$$
\ln \left(\frac{p_{1}^{*}}{\mathrm{P}_{c 1}}\right)=\frac{a_{1} \tau+a_{2} \tau^{1.5}+a_{3} \tau^{3}+a_{4} \tau^{3.5}+a_{5} \tau^{4}+a_{6} \tau^{7.5}}{1-\tau}
$$

Donde $a_{1}=7.85951783, a_{2}=1.84408259, a_{3}=-11.7866497$, $a_{4}=22.6807411, a_{5}=-15.9618719, a_{6}=1.80122502, \mathrm{P}_{C l}$ es la presión crítica del solvente aceptada por la IAPWS en 1995 $\left(\mathrm{P}_{C l}=220.64\right.$ bar $)$. Los datos experimentales de solubilidad de sulfuro de hidrógeno en agua fueron obtenidos de Lee y Mather (1977). Los valores de la presión crítica, la temperatura crítica y factor acéntrico para el sulfuro de hidrógeno se tomaron de la literatura (Duan et al., 1996) y son 89.63 bar, $373.4 \mathrm{~K}$ y 0.09 , respectivamente. El cálculo de la solubilidad del $\mathrm{H}_{2} \mathrm{~S}$ en $\mathrm{H}_{2} \mathrm{O}$ se hizo para diferentes condiciones de temperatura, presión y concentración de $\mathrm{NaC} 1$. En la Figura 1 se comparan los resultados calculados con los datos experimentales de Lee y Mather (1977). Esta figura muestra que las predicciones se encuentran aproximadamente dentro de la incertidumbre experimental (desviación máxima del 17\%) cuando se asume un valor medio del volumen molar parcial del $\mathrm{H}_{2} \mathrm{~S}$ en el cálculo del factor de corrección Poynting (Pérez et al., 2009).

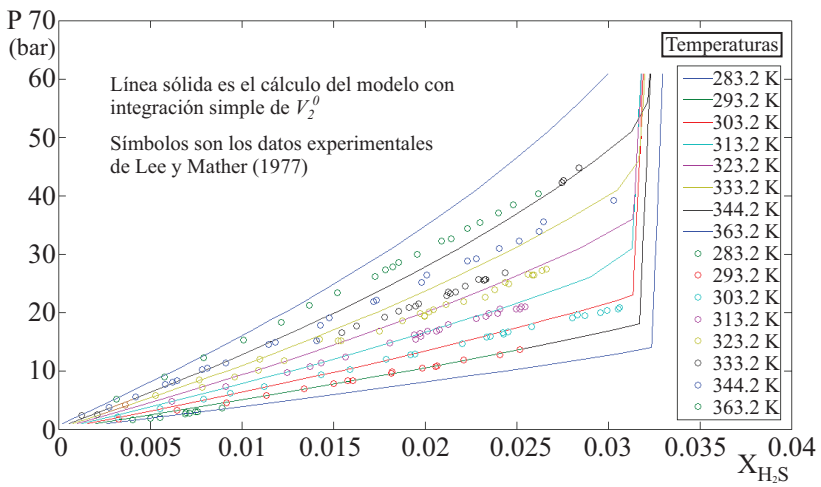

Figura 1. Comparación de la solubilidad experimental del $\mathrm{H}_{2} \mathrm{~S}$ con la predicción del modelo termodinámico empleando integración simple para $V_{2}^{0}$, a las temperaturas indicadas.

\section{El Modelo Termodinámico con Integración Analítica de $V_{2}^{0}$}

Para describir de manera más exacta el equilibrio líquido-vapor del sistema $\mathrm{H}_{2} \mathrm{~S}-\mathrm{H}_{2} \mathrm{O}-\mathrm{NaCl}$ se considera la dependencia del volumen molar parcial del soluto sobre la presión y la temperatura. En este caso, nuevamente se emplea el modelo termodinámico descrito en la sección anterior. La ecuación (11) también puede expresarse como:

$$
\ln \left(f_{2 b}\right)=\ln \left(H_{2 w} \gamma_{2 b} \chi_{2 b}\right)+\left(S_{2} m_{k}\right)+\frac{1}{R T} \int_{P_{s a t}}^{P} V_{2}^{0} d P
$$

El factor de corrección Poynting de (16) se calcula 
de acuerdo a las ecuaciones (8) y (9). El comportamiento del sistema H2S-H2O-NaCl calculado de esta manera se muestra en la Figura 2:

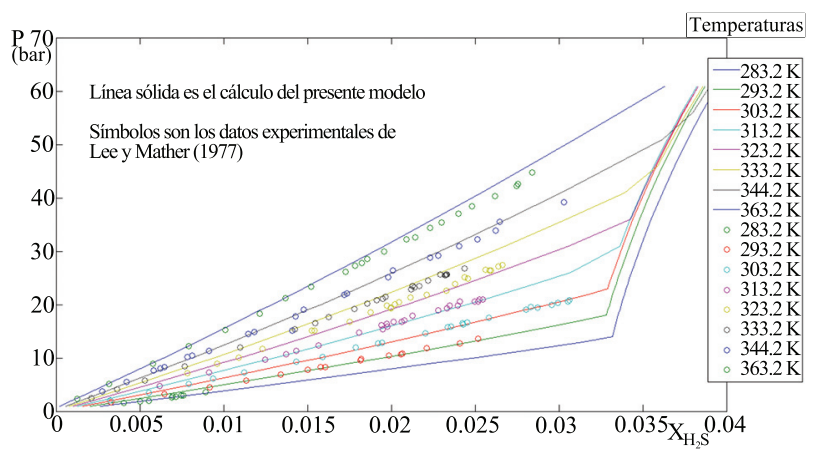

Figura 2. Comparación de la solubilidad experimental del $\mathrm{H}_{2} \mathrm{~S}$ con la predicción del presente modelo termodinámico empleando integración analítica para $V_{2}^{0}$, a las temperaturas indicadas.

Comparando las figuras 1 y 2 , se observa que al incluir la dependencia del volumen molar sobre la presión en el factor de corrección Poynting, de acuerdo a las ecuaciones (1), (8) y (9), se reproducen mejor los datos experimentales de solubilidad para el sistema $\mathrm{H}_{2} \mathrm{~S}-\mathrm{H}_{2} \mathrm{O}-\mathrm{NaCl}$ (desviación máxima del 7\%).

Partiendo de las ecuaciones anteriormente mencionadas, el volumen molar parcial a dilución infinita de un soluto gaseoso en una solución acuosa $V_{2}^{0}$, puede calcularse de la siguiente forma (Prausnitz et al., 1999; Pérez et al., 2009):

$$
\begin{gathered}
\left(\frac{\partial G_{2}^{0}}{\partial P}\right)_{T, n_{i}}=V_{2}^{0}=V_{21}^{0}+V_{22}^{0}+V_{23}^{0}+V_{24}^{0} \\
V_{21}^{0}=\frac{N v_{0}\left(P_{\text {sat }}+B\right)^{A}}{(P+B)^{A}} \\
V_{22}^{0}=\frac{A R T(1-N)}{P+B} \\
V_{23}^{0}=2 R T \Omega\left(\beta_{12}-N \beta_{11}\right) \frac{A(P+B)^{A-1}}{v_{0}\left(P_{\text {sat }}+B\right)^{A}} \exp \left[-\frac{C_{1}(P+B)^{A}}{v_{0}\left(P_{\text {sat }}+B\right)^{A}}\right] \\
V_{24}^{0}=\left(\frac{a}{T^{5}}+b\right)\left[\frac{C_{2}(P+B)^{A}}{v_{0}\left(P_{s a t}+B\right)^{A}}+\frac{C_{2}^{2}(P+B)^{2 A}}{2 v_{0}^{2}\left(P_{\text {sat }}+B\right)^{2 A}}+\frac{C_{2}^{3}(P+B)^{3 A}}{6 v_{0}^{3}\left(P_{\text {sat }}+B\right)^{3 A}}\right]
\end{gathered}
$$

Las ecuaciones (17a)-(17e) son el modelo de Plyasunov et al. (2000b) expresado en función de las correlaciones auxiliares (ecuaciones 4 a 7 ).

Empleando las ecuaciones (17a)-(17e) para el cálculo de $V_{2}^{0}$ (modelo del presente trabajo), así como la expresión de Plyasunov et al. (2000b) y los datos experimentales de Hnedkovsky et al. (1996) para el volumen molar parcial a dilución infinita $\left(V_{2}^{0}\right)$ del $\mathrm{H}_{2} \mathrm{~S}$, se obtienen gráficas de valores de $V_{2}^{0}$ de las fuentes indicadas, donde se reafirma la excelente correlación con datos experimentales que proporciona la expresión alternativa para $V_{2}^{0}$ propuesta en el presente trabajo (Figura 3, Figura 4).

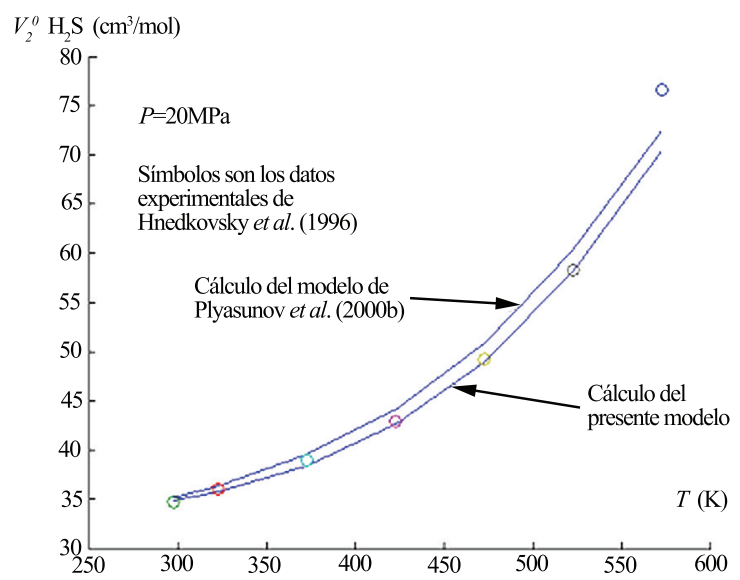

Figura 3. Comparación de los valores experimentales de $V_{2}^{0}$ del $\mathrm{H}_{2} \mathrm{~S}$ de Hnedkovsky et al. (1996) a diferentes temperaturas, con las predicciones tanto del presente modelo como con la ecuación de Plyasunov et al. (2000b) a $P=20 \mathrm{MPa}$.

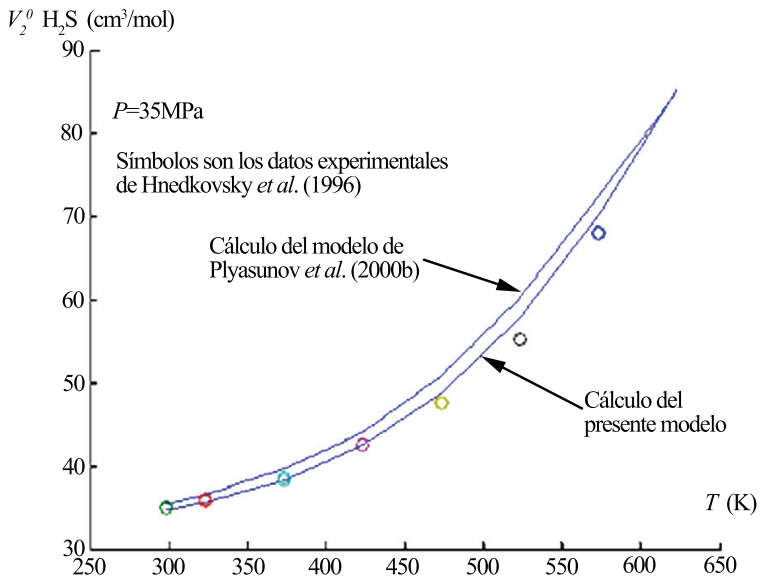

Figura 4: Comparación de los valores experimentales de $V_{2}^{0}$ del $\mathrm{H}_{2} \mathrm{~S}$ de Hnedkovsky et al. (1996) a diferentes temperaturas, con las predicciones tanto del presente modelo como con la ecuación de Plyasunov et al. (2000b) a $P=35 \mathrm{MPa}$.

De esta forma, se demuestra la consistencia matemática y conceptual de la ecuación para corrección Poynting y de la expresión alternativa para cálculo de $V_{2}^{0}$, propuestas en este manuscrito.

El presente trabajo es una propuesta original, que innova totalmente de la manera descrita. El único antecedente de una ecuación para corrección Poynting existente es una expresión muy sencilla, representada en los términos de la extrema derecha de las ecuaciones (11) y (12) (Carroll, 1991; Fernández-Prini et al., 2003). 


\section{Conclusiones}

En el presente trabajo se ha propuesto una ecuación para el cálculo del cambio isotérmico de energía estándar (dilución infinita) de Gibbs del soluto gaseoso no polar, aplicable en un intervalo de temperatura de $273.16 \mathrm{~K}$ a $647 \mathrm{~K}$, valores de presión de hasta 2 kbar y en salmueras con fuerza iónica de hasta $6 \mathrm{~m} \mathrm{NaCl}$, para aplicación de la corrección Poynting. Esta fórmula se obtiene integrando analíticamente respecto a la presión la expresión semiteórica del volumen molar parcial a dilución infinita acuosa para solutos volátiles no electrolíticos de Plyasunov et al. (2000b). La ecuación propuesta se ha aplicado en el cálculo de equilibrio de fases líquido-vapor del sistema $\mathrm{H}_{2} \mathrm{~S}-\mathrm{H}_{2} \mathrm{O}$ $\mathrm{NaCl}$, para el cual proporciona una excelente correlación con los datos experimentales (con desviación máxima del $7 \%$ ). De igual manera, se propone una expresión alternativa para calculo de $V_{2}^{0}$ (con valores semejantes de desviación máxima).

\section{Agradecimientos}

El presente trabajo fue elaborado con soporte económico del Consejo Nacional de Ciencia y Tecnología (CONACYT), al cual se expresa el agradecimiento respectivo.

\section{Referencias}

Arfken, G., Weber J.H., 1985, Mathematical Methods for Physicists: Orlando, Florida, E.U.A., Academic Press, 120 p.

Carroll, J.J., 1991, What is Henry's Law?: Chemical Engineering Progress, 87, 48-52.

Duan, Z., Moller, N., Weare, J.H., 1996, Prediction of the solubility of $\mathrm{H}_{2} \mathrm{~S}$ in $\mathrm{NaCl}$ aqueous solution: an equation of state approach: Chemical Geology, 130, 15-20.

Fernández-Prini, R., Alvarez, J.L., Harvey, A.H., 2003, Henry's constants and vapor-liquid distribution constants for gaseous solutes in $\mathrm{H}_{2} \mathrm{O}$ and $\mathrm{D}_{2} \mathrm{O}$ at high temperatures: Journal of Physical and Chemical Reference Data, 32, 903-916.

Fernández-Prini, R., Crovetto, R., 1989, Evaluation of Data on Solubility of Simple Apolar Gases in Light and Heavy Water at High Temperature: Journal of Physical and Chemical Reference Data, 18, 1231-1243.

Haas, J.L., 1970, An equation for the density of vapor-saturated $\mathrm{NaCl}-\mathrm{H}_{2} \mathrm{O}$ solutions from 75 degrees to 325 degrees C: American Journal of Science, 269, 489-493.

Harvey, A.H., 1996, Semiempirical correlation for Henry's constants over large temperature ranges: American Institute of Chemical Engineers Journal, 42, 1491-1494.

Heidemann, R.A., Jeje, A.A., Mohtadi, F., 1984, An introduction to the properties of fluids and solids: Calgary, Alberta, Canada, University of Calgary Press, $401 \mathrm{p}$.

Hnedkovsky, L., Wood, R.H., Majer, V., 1996, Volumes of aqueous solutions of $\mathrm{CH}_{4}, \mathrm{CO}_{2}, \mathrm{H}_{2} \mathrm{~S}$ and $\mathrm{NH}_{3}$ at temperatures from 298.15 to $705 \mathrm{~K}$ and pressures to $35 \mathrm{MPa}$ : The Journal of Chemical Thermodynamics, 28, 125-142.

IAPWS (International Association for the Properties of Water and Steam), 1995, Release on Values of Temperature, Pressure and Density of Ordinary and Heavy Water Substances at Their Respective Critical Points, in White, H.J., Sengers, J.V., Neumann, D.B., Bellows, J.C. (eds.), Physical Chemistry of Aqueous Systems: Meeting the Needs of Industry: New York, Begell House, A101.

Kell, G.S., 1975, Density, Thermal Expansivity, and Compressibility of liquid water from $0^{\circ}$ to $150^{\circ} \mathrm{C}$ : Correlations and Tables for atmospheric pressure and saturation reviewed and expressed on 1968 temperature scale: Journal of Chemical and Engineering Data, 20, 97-105.

Kirkwood J.G., Buff, F.P., 1951, The statistical mechanical theory of solutions I: Journal of Chemical Physics, 19, 774-777.

Knopp, K., 1996, Theory of Functions Part I and II, Two Volumes Bound as One: New York, Dover, $320 \mathrm{p}$.

Korn, G.A., Korn, T.M., 1968, Mathematical Handbook for Scientists and Engineers: New York, McGraw-Hill, 1152 p.

Krantz, S.G., 1999, Handbook of Complex Variables: Boston, Massachusetts, Birkhäuser, $290 \mathrm{p}$.

Lee, J.I., Mather, A.E., 1977, Solubility of hydrogen sulfide in water: Berichte der Bunsen-Gesellschaft für Physikalische Chemie, 81, 1021-23.

Mathews, J.H., Howell, R.W., 2006, Complex Analysis for Mathematics and Engineering: Sudbury, Massachusetts, Jones and Bartlett, 608 p.

Morse, P.M., Feshbach, H., 1953, Methods of Theoretical Physics, Part I: New York, McGraw-Hill, $657 \mathrm{p}$.

O'Connell, J.P., 1971, Thermodynamic properties of solutions based on correlation functions: Molecular Physics, 20, 27-33.

O'Connell, J.P., 1994, Thermodynamics and fluctuation solution theory with some applications to systems at near- or supercritical conditions, in Kiran, E., Levelt Sanders, J.M.H. (eds.), Supercritical Fluids Fundamentals and Applications: Dordrecht, Netherlands, Kluwer Academic Publishers, 191-229.

O'Connell, J.P., 1995, Application of fluctuation solution theory to thermodynamic properties of solutions: Fluid Phase Equilibria, 104, 21-39.

Orbey, H., Sandler, S.I., 1998, Modeling Vapor-Liquid Equilibria: cubic equations of state and their mixing rules: Cambridge, Massachusetts, Cambridge University Press, $207 \mathrm{p}$.

Perez, R.,J., Heidemann, R.A., 2006, Coupling an equation of state and Henry's law to model the phase equilibria of gases and brines: examples in the $\mathrm{N}_{2}-\mathrm{H}_{2} \mathrm{O}-\mathrm{NaCl}$ system: Journal of Geochemical Exploration, 89, 331-334.

Pérez, R.J., Heidemann, R. A., González-Partida, E., 2009, Modelo teórico para el cálculo de las solubilidades mutuas entre gases nopolares y agua con sales disueltas, Parte I: desarrollo de ecuaciones termodinámicas, Boletín de la Sociedad Geológica Mexicana, 61, 325-337.

Pitzer K.S., 1995, Thermodynamics: New York, McGraw-Hill, 626 p.

Plyasunov, A.V., O'Connell, J.P., Wood, R.H., 2000a, Infinite dilution partial molar properties of aqueous solutions of nonelectrolytes I. Equations for partial molar volumes at infinite dilution and standard thermodynamic functions of hydration of volatile nonelectrolytes over wide ranges of conditions: Geochimica et Cosmochimica Acta, $64,495-512$.

Plyasunov, A.V., O’Connell, J.P., Wood, R.H., Shock, E.L., 2000b, Infinite dilution partial molar properties of aqueous solutions of nonelectrolytes II. Equations for the standard thermodynamic functions of hydration of volatile nonelectrolytes over wide ranges of conditions including subcritical temperatures: Geochimica et Cosmochimica Acta, 64, 2779-2795.

Prausnitz, J.M., Lichtenhaler, R.N., Gomes, E., 1999, Molecular Thermodynamics of Fluid Phase Equilibria: New Jersey, Prentice Hall, 860 p.

Rowlinson, J.S., Swinton, F.L., 1982, Liquids and liquid mixtures: London, Butterworth Scientific, $328 \mathrm{p}$.

Saul, A., Wagner, W., 1987, International Equations for the saturation properties of ordinary water substance: Journal of Physical and Chemical Reference Data, 16, 893-901.

Setchenow, M., 1892, Action de l'acide carbonique sur les solutions des sels a acides forts: Annales de Chimie et de Physique, 25, 226-270.

Soave, G., 1972, Equilibrium constants from a modified Redlich-Kwong equation of state: Chemical Engineering Science, 27, 1197-1203. 
Wagner, W., Pruss, A., 1993, International equations for the saturation properties of ordinary water substance. Revised according to the International Temperature Scale of 1990. Addendum to Journal of Physical and Chemical Reference Data 16: Journal of Physical and Chemical Reference Data, 22, 783-787.

\section{Anexo A: Integración del Término $\Delta G_{23}{ }^{0}$}

$$
\begin{gathered}
\left(-\frac{1}{C_{1}}\right) \int_{P_{\text {sat }}}^{P}\left[-\frac{C_{1} A}{v_{0}\left(P_{\text {sat }}+B\right)^{A}}\right](P+B)^{A-1} \exp \left[-\frac{C_{1}}{v_{0}\left(P_{\text {sat }}+B\right)^{A}}(P+B)^{A}\right] d P \\
\int_{P_{\text {sat }}}^{P} e^{U} d U=\left[e^{U}\right]_{P_{\text {sat }}}^{P} \\
U=-\frac{C_{1}}{v_{0}\left(P_{\text {sat }}+B\right)^{A}}(P+B)^{A} \\
d U=\left[-\frac{C_{1} A}{v_{0}\left(P_{\text {sat }}+B\right)^{A}}\right](P+B)^{A-1} d P \\
=-\frac{1}{C_{1}} \exp \left[-\frac{C_{1}}{v_{0}\left(P_{\text {sat }}+B\right)^{A}}(P+B)^{A}\right]_{P_{\text {sat }}}^{P} \\
=-\frac{1}{C_{1}} \exp \left[-\frac{C_{1}}{v_{0}\left(P_{\text {sat }}+B\right)^{A}}(P+B)^{A}\right]-\left\{-\frac{1}{C_{1}} \exp \left[-\frac{C_{1}}{v_{0}\left(P_{\text {sat }}+B\right)^{A}}\left(P_{\text {sat }}+B\right)^{A}\right]\right\} \\
(\mathrm{A} 6) \\
=\frac{1}{C_{1}}\left\{\exp \left[-\frac{C_{1}}{v_{0}}\right]-\exp \left[-\frac{C_{1}(P+B)^{A}}{v_{0}\left(P_{\text {sat }}+B\right)^{A}}\right]\right\} \\
=\frac{1}{C_{1}}\left\{\exp \left[-\frac{C_{1}}{v_{0}}\right]-\exp \left[-C_{1} \rho_{P, T}\right]\right\}
\end{gathered}
$$

\section{Anexo B: Integración del Término $\Delta G_{24}{ }^{0}$}

$$
\begin{gathered}
\int_{P_{s a t}}^{P} \exp \left[\frac{C_{2}}{v_{0}\left(P_{\text {sat }}+B\right)^{A}}(P+B)^{A}\right] d P \\
\mathrm{Si} f(P)=\exp \left[\frac{C_{2}}{v_{0}\left(P_{\text {sat }}+B\right)^{A}}(P+B)^{A}\right]
\end{gathered}
$$

Haciendo el cambio de variable:

$$
\begin{array}{r}
x=\frac{C_{2}}{v_{0}\left(P_{\text {sat }}+B\right)^{A}}(P+B)^{A} \\
\therefore f(x)=e^{x}
\end{array}
$$

Con aproximación polinomial:

$$
f(x)=e^{x}=1+x+\frac{x^{2}}{2 !}+\frac{x^{3}}{3 !}+\cdots+\frac{x^{n}}{n !}
$$

Usando los 4 primeros términos de la aproximación polinomial:

$$
f(x)=e^{x}=1+x+\frac{x^{2}}{2}+\frac{x^{3}}{6}
$$

Sustituyendo la ecuación (B3) en la ecuación (B6) se obtiene la siguiente expresión:

$f(P)=1+\frac{C_{2}}{v_{0}\left(P_{\text {sat }}+B\right)^{A}}(P+B)^{A}+\frac{c_{2}^{2}}{2 v_{0}^{2}\left(P_{\text {sat }}+B\right)^{2 A}}(P+B)^{2 A}+\frac{C_{2}^{3}}{6 v_{0}^{3}\left(P_{\text {sat }}+B\right)^{3 A}}(P+B)^{3 A}$
Manuscrito recibido: Febrero 8, 2010

Manuscrito corregido recibido: Abril 12, 2010.

Manuscrito aceptado: Mayo 4, 2010.

(Morse y Feshback, 1953; Korn y Korn, 1968; Arfken y Weber, 1985; Knopp, 1996; Krantz, 1999; Mathews y Howell, 2006).

$$
\begin{aligned}
& \therefore \int_{P_{\text {sat }}}^{P} \exp \left[\frac{C_{2}}{v_{0}\left(P_{\text {sat }}+B\right)^{A}}(P+B)^{A}\right] d P= \\
& =\int_{P_{\text {sat }}}^{p}\left[1+\frac{C_{2}}{v_{0}\left(P_{\text {sat }}+B\right)^{A}}(P+B)^{A}+\frac{C_{2}^{2}}{2 v_{0}^{2}\left(P_{\text {sat }}+B\right)^{2 A}}(P+B)^{2 A} \ldots\right. \\
& \left.+\frac{C_{2}^{3}}{6 v_{0}^{3}\left(P_{\text {sat }}+B\right)^{3 A}}(P+B)^{3 A}\right] d P \\
& \int_{P_{\text {sat }}}^{P} d P+\frac{C_{2}}{v_{0}\left(P_{\text {sat }}+B\right)^{A}} \int_{P_{\text {sat }}}^{P}(P+B)^{A} d P \ldots \\
& +\frac{C_{2}^{2}}{2 v_{0}^{2}\left(P_{\text {sat }}+B\right)^{2 A}} \int_{P_{\text {sat }}}^{P}(P+B)^{2 A} d P+\frac{C_{2}^{3}}{6 v_{0}^{3}\left(P_{\text {sat }}+B\right)^{3 A}} \int_{P_{\text {sat }}}^{P}(P+B)^{3 A} d P \\
& =\left[P+\frac{C_{2}}{v_{0}\left(P_{\text {sat }}+B\right)^{A}} \frac{(P+B)^{A+1}}{(A+1)}+\frac{C_{2}^{2}}{2 v_{0}^{2}\left(P_{\text {sat }}+B\right)^{2 A}} \frac{(P+B)^{2 A+1}}{(2 A+1)}\right. \\
& \left.+\frac{C_{2}^{3}}{6 v_{0}^{3}\left(P_{\text {sat }}+B\right)^{3 A}} \frac{(P+B)^{3 A+1}}{(3 A+1)}\right]_{P_{\text {sat }}}^{P}
\end{aligned}
$$

$$
\begin{gathered}
=\left[P+\frac{C_{2}(P+B)^{A+1}}{v_{0}\left(P_{\text {sat }}+B\right)^{A}(A+1)}+\frac{C_{2}^{2}(P+B)^{2 A+1}}{2 v_{0}^{2}\left(P_{\text {sat }}+B\right)^{2 A}(2 A+1)} \cdots\right. \\
\left.+\frac{C_{2}^{3}(P+B)^{3 A+1}}{6 v_{0}^{3}\left(P_{\text {sat }}+B\right)^{3 A}(3 A+1)}\right]-\cdots
\end{gathered}
$$$$
-\left[P_{\text {sat }}+\frac{C_{2}\left(P_{\text {sat }}+B\right)^{A+1}}{v_{0}\left(P_{\text {sat }}+B\right)^{A}(A+1)}+\frac{C_{2}^{2}\left(P_{\text {sat }}+B\right)^{2 A+1}}{2 v_{0}^{2}\left(P_{\text {sat }}+B\right)^{2 A}(2 A+1)}+\frac{C_{2}^{3}\left(P_{\text {sat }}+B\right)^{3 A+1}}{6 v_{0}^{3}\left(P_{\text {sat }}+B\right)^{3 A}(3 A+1)}\right]
$$

$$
=P-P_{\text {sat }}+\frac{C_{2}}{v_{0}\left(P_{\text {sat }}+B\right)^{A}(A+1)}\left[(P+B)^{A+1}-\left(P_{\text {sat }}+B\right)^{A+1}\right]+
$$

$$
\frac{C_{2}^{2}}{2 v_{0}^{2}\left(P_{\text {sat }}+B\right)^{2 A}(2 A+1)}\left[(P+B)^{2 A+1}-\left(P_{\text {sat }}+B\right)^{2 A+1}\right]+
$$$$
\frac{C_{2}^{3}}{6 v_{0}^{3}\left(P_{\text {sat }}+B\right)^{3 A}(3 A+1)}\left[(P+B)^{3 A+1}-\left(P_{\text {sat }}+B\right)^{3 A+1}\right]
$$

\section{Anexo C: Determinación detallada de $V_{2}^{0}$ a partir de $\Delta G_{2}^{0}$}

El volumen molar parcial a dilución infinita de un soluto gaseoso en una mezcla gaseosa puede calcularse como:

$$
V_{2}^{0}=\left(\frac{\partial G_{2}^{0}}{\partial P}\right)_{T, n_{i}}
$$


Derivando respecto a la presión las anteriores expresiones:

$$
\begin{aligned}
& \frac{\partial G_{21}^{0}}{\partial P}=\frac{\partial}{\partial P}\left\{\frac{N v_{0}\left(P_{\text {sat }}+B\right)^{A}}{1-A}\left[\frac{1}{(P+B)^{A-1}}-\frac{1}{\left(P_{\text {sat }}+B\right)^{A-1}}\right]\right\} \\
& =\frac{N v_{0}\left(P_{\text {sat }}+B\right)^{A}}{1-A} \frac{\partial(P+B)^{1-A}}{\partial P}=\frac{N v_{0}\left(P_{\text {sat }}+B\right)^{A}}{1-A}(1-A)(P+B)^{1-A-1} \\
& =N v_{0}\left(P_{\text {sat }}+B\right)^{A}(P+B)^{-A}=\frac{N v_{0}\left(P_{\text {sat }}+B\right)^{A}}{(P+B)^{A}} \\
& \frac{\partial G_{22}^{0}}{\partial P}=\frac{\partial}{\partial P}\left\{R T(1-N) \ln \left[\frac{P+B}{P_{\text {sat }}+B}\right]^{A}\right\} \\
& =R T(1-N) \frac{1}{\left[\frac{P+B}{P_{\text {sat }}+B}\right]^{A}} \frac{\partial}{\partial P}\left[\frac{P+B}{P_{\text {sat }}+B}\right]^{A} \\
& =R T(1-N)\left[\frac{P_{\text {sat }}+B}{P+B}\right]^{A} \frac{1}{\left(P_{\text {sat }}+B\right)^{A}} A(P+B)^{A-1} \\
& =R T(1-N) \frac{A(P+B)^{A-1}}{(P+B)^{A}}=\frac{A R T(1-N)}{P+B} \\
& \frac{\partial G_{23}^{0}}{\partial P}=\frac{\partial}{\partial P}\left\{2 R T \Omega\left(\beta_{12}-N \beta_{11}\right)\left(\frac{1}{C_{1}}\right)\left\langle\exp \left[-\frac{C_{1}}{v_{0}}\right]-\exp \left[-\frac{C_{1}(P+B)^{A}}{v_{0}\left(P_{\text {sat }}+B\right)^{A}}\right]\right\rangle\right\} \\
& =2 R T \Omega\left(\beta_{12}-N \beta_{11}\right) \frac{1}{C_{1}} \frac{C_{1}}{v_{0}\left(P_{s a t}+B\right)^{A}} \frac{\partial(P+B)^{A}}{\partial P} \exp \left[-\frac{C_{1}(P+B)^{A}}{v_{0}\left(P_{\text {sat }}+B\right)^{A}}\right] \\
& =2 R T \Omega\left(\beta_{12}-N \beta_{11}\right) \frac{A(P+B)^{A-1}}{v_{0}\left(P_{\text {sat }}+B\right)^{A}} \exp \left[-\frac{C_{1}(P+B)^{A}}{v_{0}\left(P_{\text {sat }}+B\right)^{A}}\right]
\end{aligned}
$$$$
=\left(\frac{a}{T^{5}}+b\right)\left[\frac{C_{2}(P+B)^{A}}{v_{0}\left(P_{\text {sat }}+B\right)^{A}}+\frac{C_{2}^{2}(P+B)^{2 A}}{2 v_{0}^{2}\left(P_{\text {sat }}+B\right)^{2 A}}+\frac{C_{2}^{3}(P+B)^{3 A}}{6 v_{0}^{3}\left(P_{\text {sat }}+B\right)^{3 A}}\right]
$$

$$
=\left(\frac{a}{T^{5}}+b\right)\left[\frac{C_{2}(A+1)(P+B)^{A}}{v_{0}\left(P_{\text {sat }}+B\right)^{A}(A+1)}+\frac{C_{2}^{2}(2 A+1)(P+B)^{2 A}}{2 v_{0}^{2}\left(P_{\text {sat }}+B\right)^{2 A}(2 A+1)}+\frac{C_{2}^{3}(3 A+1)(P+B)^{3 A}}{6 v_{0}^{3}\left(P_{\text {sat }}+B\right)^{3 A}(3 A+1)}\right]
$$

Resumen:

$$
\left(\frac{\partial G_{2}^{0}}{\partial P}\right)_{T, n_{i}}=V_{2}^{0}=V_{21}^{0}+V_{22}^{0}+V_{23}^{0}+V_{24}^{0}
$$

$$
\begin{gathered}
V_{21}^{0}=\frac{N v_{0}\left(P_{\text {sat }}+B\right)^{A}}{(P+B)^{A}} \\
V_{22}^{0}=\frac{A R T(1-N)}{P+B} \\
V_{23}^{0}=2 R T \Omega\left(\beta_{12}-N \beta_{11}\right) \frac{A(P+B)^{A-1}}{v_{0}\left(P_{\text {sat }}+B\right)^{A}} \exp \left[-\frac{C_{1}(P+B)^{A}}{v_{0}\left(P_{\text {sat }}+B\right)^{A}}\right] \\
V_{24}^{0}=\left(\frac{a}{T^{5}}+b\right)\left[\frac{C_{2}(P+B)^{A}}{v_{0}\left(P_{\text {sat }}+B\right)^{A}}+\frac{C_{2}^{2}(P+B)^{2 A}}{2 v_{0}^{2}\left(P_{\text {sat }}+B\right)^{2 A}}+\frac{C_{2}^{3}(P+B)^{3 A}}{6 v_{0}^{3}\left(P_{\text {sat }}+B\right)^{3 A}}\right]
\end{gathered}
$$

